\title{
Why Post-starburst Galaxies Are Now Quiescent
}

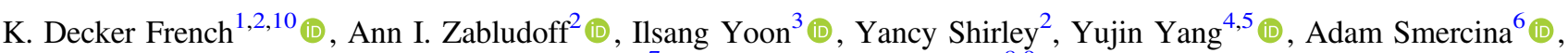 \\ J. D. Smith ${ }^{7}$ (D), and Desika Narayanan ${ }^{8,9}$ (iD) \\ ${ }^{1}$ Observatories of the Carnegie Institute for Science, 813 Santa Barbara Street, Pasadena, CA 91101, USA \\ ${ }^{2}$ Steward Observatory, University of Arizona, 933 North Cherry Avenue, Tucson, AZ 85721, USA \\ ${ }^{3}$ National Radio Astronomy Observatory, 520 Edgemont Road, Charlottesville, VA 22903, USA \\ ${ }^{4}$ Korea Astronomy and Space Science Institute, 776 Daedeokdae-ro, Yuseong-gu, Daejeon, 305-348, Republic of Korea \\ ${ }^{5}$ Korea University of Science and Technology (UST), 217 Gajeong-ro Yuseong-gu, Daejeon, 34113, Republic of Korea \\ ${ }^{6}$ Department of Astronomy, University of Michigan, 1085 S. University Avenue, Ann Arbor, MI 48109, USA \\ ${ }^{7}$ Department of Physics and Astronomy, University of Toledo, Ritter Obs., MS \#113, Toledo, OH 43606, USA \\ ${ }^{8}$ Department of Astronomy, University of Florida, 211 Bryant Space Sciences Center, Gainesville, FL 32611, USA \\ ${ }^{9}$ Cosmic Dawn Center (DAWN), Niels Bohr Institute, University of Copenhagen, Juliane Maries vej 30, DK-2100 Copenhagen, Denmark \\ Received 2018 February 20; revised 2018 May 18; accepted 2018 May 28; published 2018 July 12
}

\begin{abstract}
Post-starburst or "E + A" galaxies are rapidly transitioning from star-forming to quiescence. While the current star formation rate (SFR) of post-starbursts is already at the level of early-type galaxies, we recently discovered that many have large CO-traced molecular gas reservoirs consistent with normal star-forming galaxies. These observations raise the question of why these galaxies have such low SFRs. Here we present an ALMA search for the denser gas traced by $\mathrm{HCN}(1-0)$ and $\mathrm{HCO}^{+}(1-0)$ in two CO-luminous, quiescent post-starburst galaxies. Intriguingly, we fail to detect either molecule. The upper limits are consistent with the low SFRs and with earlytype galaxies. The $\mathrm{HCN} / \mathrm{CO}$ luminosity ratio upper limits are low compared to star-forming and even many earlytype galaxies. This implied low dense gas mass fraction explains the low SFRs relative to the CO-traced molecular gas and suggests that the state of the gas in post-starburst galaxies is unusual, with some mechanism inhibiting its collapse to denser states. We conclude that post-starbursts galaxies are now quiescent because little dense gas is available, in contrast to the significant CO-traced lower density gas reservoirs that still remain.
\end{abstract}

Key words: galaxies: evolution - galaxies: ISM

\section{Introduction}

The nature of how and when the molecular gas reservoirs are depleted in galaxies is essential to understanding the question of why galaxies become quiescent. To explore this question, we focus on a class of galaxies in the midst of rapid evolution in their star formation properties. The spectra of post-starburst, "E + A," or " $\mathrm{k}+\mathrm{a}$ " galaxies show strong Balmer absorption, which is indicative of a recent starburst that ended in the last gigayear, yet little ongoing star formation, indicating a rapid change from star-forming to quiescent (Dressler \& Gunn 1983; Couch \& Sharples 1987). Despite their low star formation rates (SFRs), many (over half of those studied) have large CO-traced molecular gas reservoirs (French et al. 2015; Rowlands et al. 2015; Alatalo et al. 2016c). These quiescent post-starburst galaxies have similar CO-traced molecular gas fractions $\left(M_{\mathrm{mol}} / M_{\star}\right)$ as normal star-forming galaxies, implying a lower CO-traced star formation efficiency SFE $\propto$ SFR $/ L^{\prime}(\mathrm{CO})$. This offset persists in the classical Kennicutt-Schmidt (Kennicutt 1998) relation, suggesting that post-starburst galaxies experience a $\sim 4 \times$ suppression of SFE in the CO-traced molecular gas.

Previous studies of the molecular gas content of poststarburst galaxies have used the $\mathrm{CO}(1-0)$ and $\mathrm{CO}(2-1)$ lines as tracers. The $\mathrm{CO}(1-0)$ line is sensitive to molecular gas at densities $\sim 100 \mathrm{~cm}^{-3}$. Other molecules, such as HCN (1-0) and $\mathrm{HCO}^{+}(1-0)$, trace denser gas. The critical densities of $\mathrm{HCN}(1-0)$ and $\mathrm{HCO}^{+}(1-0)$ are $3 \times 10^{6} \mathrm{~cm}^{-3}$ and $2 \times$ $10^{5} \mathrm{~cm}^{-3}$ (e.g., Juneau et al. 2009), though they are also sensitive to less dense gas, with effective excitation densities

\footnotetext{
${ }^{10}$ Hubble Fellow.
}

of $8.4 \times 10^{3} \mathrm{~cm}^{-3}$ and $950 \mathrm{~cm}^{-3}$, respectively (Shirley 2015). The HCN (1-0) luminosity correlates more linearly with SFR, and with less scatter, than does the CO (1-0) luminosity (Gao \& Solomon 2004), even down to the scales of starforming clumps (Wu et al. 2005, 2010). In starburst galaxies, which have high CO-traced SFEs, the ratio of HCN to CO luminosity is high, resulting in a high dense gas mass consistent with the high SFRs. An analogous situation may apply in the post-starburst galaxies: if we measure a low dense gas mass, this would be consistent with the low SFRs in these galaxies, despite the higher CO luminosities.

We must measure the properties of this denser gas in poststarburst galaxies to understand why there is no significant star formation and why $\mathrm{CO}$-traced molecular gas remains. Here, we present an ALMA survey of $\mathrm{HCN}(1-0)$ and $\mathrm{HCO}^{+}(1-0)$ in two CO-luminous post-starburst galaxies.

\section{Data \\ 2.1. ALMA Observations}

The observations for this work were obtained during ALMA Cycle 4 (program 2016.1.00881.S; PI: French). We observe two post-starburst galaxies with $\mathrm{CO}(1-0)$ detections from French et al. (2015) with representative CO luminosities and SFRs, and with redshifts and declinations enabling observations with ALMA (labeled H02 and S05). We use the Band 3 receiver $(84-116 \mathrm{GHz})$ with two spectral windows to observe HCN $1-0$ (88.63 GHz rest frame), $\mathrm{HCO}^{+} 1-0(89.19 \mathrm{GHz}$ rest frame), and $\mathrm{HNC} 1-0$ (90.66 GHz rest frame). The redshift of $\mathrm{H} 02$ pushes HCN close to the edge of the Band 3 bandwidth, so we adopt narrow spectral windows, of 417, 831, and 
$817 \mathrm{~km} \mathrm{~s}^{-1}$ for the lines, respectively. These spectral windows are still much larger than the $170 \mathrm{~km} \mathrm{~s}^{-1}$ linewidth of the CO (1-0) line for this galaxy. For S05, we adopt wider spectral windows of $1622-1660 \mathrm{~km} \mathrm{~s}^{-1}$.

The ALMA observations described here have a beam size of $1-1.15$ (or $1.0-1.4 \mathrm{kpc}$ ), with a maximum recoverable scale of 7 !" 6 . The beam size is chosen to match the observed CO (2-1) sizes, as our companion ALMA program (2015.1.00665.S; PI: Smith; A. Smercina et al. 2018, in preparation) finds the CO (2-1) emission confined to the central $1^{\prime \prime}-1$." 5 of these galaxies. Both the CO (2-1) sizes and Spitzer IRAC $8 \mu \mathrm{m}$ sizes are smaller than the optical sizes. The optical $r$-band half-light radii of these galaxies is 1.71 and 2 !"74 for $\mathrm{H} 02$ and S05, respectively. The resolved $\mathrm{CO}(2-1)$ fluxes are consistent with the unresolved IRAM $30 \mathrm{~m} \mathrm{CO}(2-1)$ observations (with $\sim 11^{\prime \prime}$ beam size), indicating a lack of significant $\mathrm{CO}$ emitting gas beyond the central $1-2 \mathrm{kpc}$ of these galaxies. Thus, it is unlikely that significant $\mathrm{HCN}$ emission is resolved out by our observations, as $\mathrm{HCN}$ emission is unlikely to be found outside of the $\mathrm{CO}$ emitting regions.

Additionally, interferometric observations typically constrain $\mathrm{HCN}$ emission to come from the central kiloparsec of galaxies (e.g., Aalto et al. 2012; Kepley et al. 2014; Chen et al. 2015; Scoville et al. 2015). Even when this dense gas is more extended or in outflows (e.g., Alatalo et al. 2014; Salas et al. 2014), the $\mathrm{HCN}$ emission does not extend more than $500 \mathrm{pc}$ from the main disk. The ALMA HCN observations can therefore be directly compared to the single dish CO (1-0) measurements.

The observations of $\mathrm{H} 02$ were executed in one block on 2016 November 4. The observations of S05 were executed in four blocks on 2016 November 22 and 25, and 2017 May 4. The $12 \mathrm{~m}$ array was used for both data sets, using 43 antennas for $\mathrm{H} 02$ and 41-46 antennas for S05. The observations were carried out in configurations $\mathrm{C} 40-5$ and $\mathrm{C} 40-4$ for $\mathrm{H} 02$ and S05, respectively.

The data were pipeline-calibrated using CASA version 4.7.2. The H02 observations of HCN (1-0) have a final beam size of 1 !" $0 \times 0$ ". 73 and a sensitivity of $5.5 \mathrm{mJy} /$ beam at a spectral resolution of $0.252 \mathrm{~km} \mathrm{~s}^{-1}$. The $\mathrm{H} 02$ observations of $\mathrm{HCO}^{+}$ $(1-0)$ have a final beam size of 1 !" $1 \times 0$ " 72 and a sensitivity of $2.7 \mathrm{mJy} /$ beam at a spectral resolution of $1.004 \mathrm{~km} \mathrm{~s}^{-1}$. Neither line is detected. The S05 observations of HCN (1-0) and $\mathrm{HCO}^{+}(1-0)$ are also nondetections. The S05 observations of HCN (1-0) have a final beam size of 1 !" $4 \times 1$ !" 2 and a sensitivity of $570 \mu \mathrm{Jy} /$ beam at a spectral resolution of $1.729 \mathrm{~km} \mathrm{~s}^{-1}$. The S05 observations of $\mathrm{HCO}^{+}(1-0)$ have a final beam size of 1 ."3 31 !' 2 and a sensitivity of $570 \mu \mathrm{Jy} /$ beam at a spectral resolution of $1.718 \mathrm{~km} \mathrm{~s}^{-1}$. In order to calculate upper limits on the integrated intensities, we assume the same linewidths as the CO observations: $172 \mathrm{~km} \mathrm{~s}^{-1}$ for $\mathrm{H} 02$ and $350 \mathrm{~km} \mathrm{~s}^{-1}$ for S05. The integrated HCN (1-0) intensity maps are shown in Figure 1.

We test the robustness of these upper limits in several ways. First, we taper the data to $5^{\prime \prime}$, but still do not detect either galaxy in $\mathrm{HCN}(1-0)$. Second, we use a matched filter technique (Loomis et al. 2018) using the prior information from the $\mathrm{CO}$ observations, but do not find any significant detections just below our sensitivity limit.

\subsection{Star Formation Rates}

Determining SFRs for post-starburst galaxies is complicated by the recent starburst, possible AGN activity, and heating from the young A-star population. In French et al. (2015, Section 2.5), we explored many possible methods for calculating SFRs in the post-starburst galaxies using the available archival optical and radio data. Recent work by Smercina et al. (2018) has explored a number of infrared tracers for these same galaxies. Here, we discuss the constraints on the SFRs of the two galaxies considered here from various measures.

In French et al. (2015), we compared the SFRs derived from $\mathrm{H} \alpha$ emission and the $4000 \AA$ Balmer break $\mathrm{D}_{n}(4000)$, corrected for dust based on the Balmer decrement and for aperture based on their SDSS colors. A decreased SFE in the CO-traced gas was seen for both measures, which is conservative because both SFR indicators are likely upper limits: the high incidence of LINER-like emission-line ratios in post-starbursts suggest that the $\mathrm{H} \alpha$ fluxes are likely to be contaminated, and the $\mathrm{D}_{n}(4000)$-based SFRs have greater uncertainties and trace a longer period of star formation, and are thus contaminated by the starburst itself. The SFRs derived from the extinction-corrected $\mathrm{H} \alpha$ fluxes are $0.09,0.58 M_{\odot} \mathrm{yr}^{-1}$ for $\mathrm{H} 02$ and S05 respectively. While the $\mathrm{D}_{n}(4000)$-based SFRs have significantly higher uncertainties, the $68 \%$ ranges are $0.01-0.29 M_{\odot} \mathrm{yr}^{-1}$ for $\mathrm{H} 02$ and $0.006-$ $1.05 M_{\odot} \mathrm{yr}^{-1}$ for S05, consistent with the H $\alpha$-based SFRs.

We also consider several other SFR indicators to account for the possibility that the Balmer decrement measurements underestimate the true dust obscuration. We use the VLA FIRST Survey (Faint Images of the Radio Sky at Twenty centimeters Becker et al. 1995) to study the $1.4 \mathrm{GHz}$ radio continuum emission as an SFR tracer. $1.4 \mathrm{GHz}$ emission is often used as an extinction-free tracer of SFRs (Condon 1992), but LINERs are found to contribute to the $1.4 \mathrm{GHz}$ emission (de Vries et al. 2007; Morić et al. 2010) with greater scatter than for $\mathrm{H} \alpha$ (Morić et al. 2010). Neither post-starburst galaxy considered here is detected in FIRST, and upper limits imply $1 \sigma$ upper limits on the SFRs of $<0.6$ and $0.5 M_{\odot} \mathrm{yr}^{-1}$ for $\mathrm{H} 02$ and S05, respectively.

Smercina et al. (2018) fit the dust continuum to estimate SFRs from the total infrared (TIR) flux, as well as the other infrared tracers $[\mathrm{Ne}$ II $]+[\mathrm{Ne}$ III $]$ and $[\mathrm{C} \mathrm{II}]$. S05 has a TIR SFR consistent with the other measures from the optical and radio, at $0.71 M_{\odot} \mathrm{yr}^{-1}$, and a lower SFR from [Ne II] + [Ne III] of $<0.09 M_{\odot} \mathrm{yr}^{-1}$. H02 has a significantly higher TIR SFR, at $4.6 M_{\odot} \mathrm{yr}^{-1}$. However, Smercina et al. (2018) find that the TIR flux is affected by A stellar population heating of the dust, which enhances the SFR estimate by a factor of ¿3-4×, consistent with TIR being a much longer duration SFR tracer.

The TIR flux could be an even larger overestimate of the true SFR, as Hayward et al. (2014) find that the TIR flux overestimates the true SFR by $\gtrsim 30 \times$ during the post-starburst phase. If AGN heating is important, it would also act to boost the TIR flux, causing this tracer to be an overestimate of the SFR. A TIR SFR for $\mathrm{H} 02$ of $\sim 1 M_{\odot} \mathrm{yr}^{-1}$ with the correction for A star heating would be consistent with the SFR estimated from [C II] for $\mathrm{H} 02\left(1.3 M_{\odot} \mathrm{yr}^{-1}\right)$, and the $3 \sigma$ upper limit on the $1.4 \mathrm{GHz}$ nondetection $\left(1.8 M_{\odot} \mathrm{yr}^{-1}\right)$.

Thus, the SFRs from various tracers are consistent with a range of $0.09(\mathrm{H} \alpha)-1$ (TIR) $M_{\odot} \mathrm{yr}^{-1}$ for $\mathrm{H} 02$, and $0.58 M_{\odot} \mathrm{yr}^{-1}$ or less for S05. The source of the discrepant SFRs for $\mathrm{H} 02$ between the low $\mathrm{H} \alpha$ and even $\mathrm{D}_{n}(4000)$-derived values and the high IR - derived values could be either (1) an underestimate of the extinction compared to what is measured using the Balmer 

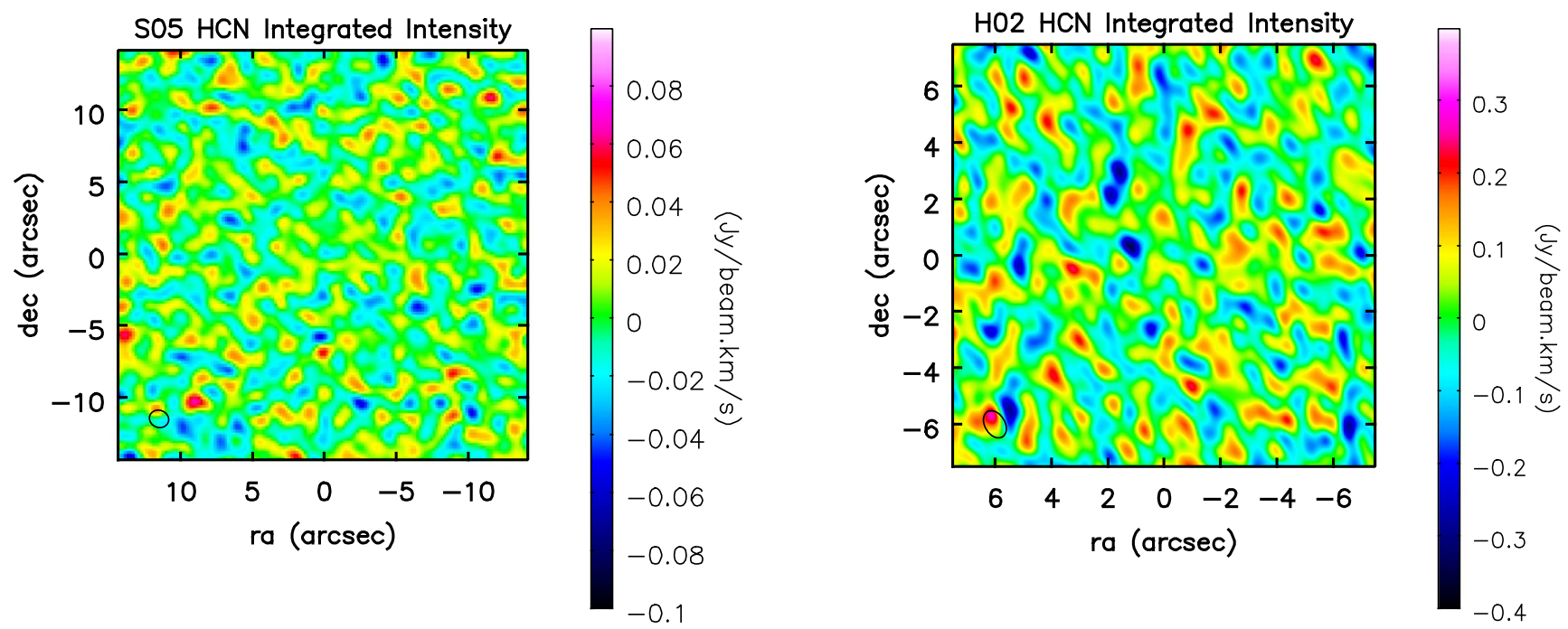

Figure 1. Integrated intensity maps for the two post-starburst targets for $\mathrm{HCN}(1-0)$. The galaxy optical centers are at $(0,0)$ on each plot. Neither source is detected.

Table 1

Post-starburst ALMA Observations

\begin{tabular}{lcccccr}
\hline \hline Name & $\begin{array}{c}\text { RA (J2000) } \\
(\mathrm{deg})\end{array}$ & $\begin{array}{c}\text { Dec }(\mathrm{J} 2000) \\
(\mathrm{deg})\end{array}$ & $z$ & $\begin{array}{c}L^{\prime}(\mathrm{CO})^{\mathrm{a}} \\
\left(10^{6} \mathrm{~K} \mathrm{~km} \mathrm{~s}^{-1} \mathrm{pc}^{2}\right)\end{array}$ & $\begin{array}{c}\mathrm{SFR}^{\mathrm{b}} \\
\left(M_{\odot} \mathrm{yr}^{-1}\right)\end{array}$ & $\begin{array}{c}L^{\prime}\left(\mathrm{HCN}^{\mathrm{c}}\right. \\
\left(10^{6} \mathrm{~K} \mathrm{~km} \mathrm{~s}^{-1} \mathrm{pc}^{2}\right)\end{array}$ \\
\hline H02 & 141.580383 & 18.678055 & 0.0541 & $842 \pm 174$ & $<1$ & $<2.3$ \\
S05 & 146.112335 & 4.499120 & 0.0467 & $304 \pm 95$ & 0.58 & $<28.9$ \\
\hline
\end{tabular}

Notes.

${ }^{\mathrm{a}}$ French et al. (2015).

${ }^{\mathrm{b}}$ From $\mathrm{H} \alpha$ flux for S05 and TIR flux for H02 (see Section 2.2).

c $3 \sigma$ upper limits.

decrement, or (2) an underestimate of the dust heating from sources other than star formation, or a combination of both. The dust masses for the two galaxies are similar $\left(\log M_{\text {dust }}=\right.$ $6.86 \pm 0.90,7.00 \pm 0.16$ for $\mathrm{H} 02$ and S05 respectively, Smercina et al. 2018), so if the extinction is much higher in $\mathrm{H} 02$, the geometry must be different. Given the uncertainties in accurately determining the dust heating contribution from AGNs or A stars, we adopt a conservative upper limit of $<1 M_{\odot} \mathrm{yr}^{-1}$ on the SFR of H02. We consider the effect of this uncertainty in the SFR of H02 in Section 3.

\section{Results}

We calculate the dense gas line luminosities using

$$
L_{\text {line }}^{\prime}=3.25 \times 10^{7}(1+z)^{-3} \nu_{\text {obs }}^{-2} S_{\text {line }} \Delta v D_{L}^{2}
$$

where $L^{\prime}$ is the line luminosity in $\mathrm{K} \mathrm{km} \mathrm{s}^{-1} \mathrm{pc}^{2}, z$ is the redshift, $\nu_{\text {obs }}$ is the observed line frequency in $\mathrm{GHz}, S_{\text {line }} \Delta v$ is the integrated flux density in $\mathrm{Jy} \mathrm{km} \mathrm{s}{ }^{-1}$, and $D_{L}$ is the luminosity distance in Mpc. We integrate over the velocity width of the CO (1-0) lines as measured using the IRAM $30 \mathrm{~m}$ (French et al. 2015). In Table 1 , we present the $3 \sigma$ upper limits on the $\mathrm{HCN}(1-0)$ and $\mathrm{HCO}^{+}(1-0)$ line luminosities for the two poststarburst targets.

We compare the $L^{\prime}(\mathrm{HCN})$ upper limits to the SFRs and CO line luminosities in Figure 2. For comparison, we also show the rest of the French et al. (2015) post-starburst sample, as well as comparison samples of star-forming and early-type galaxies with both HCN and CO measurements (Gao \& Solomon 2004;
Crocker et al. 2012). For the early-type galaxies, SFRs are from Davis et al. (2014), and we use the conversion factor from main beam temperature to flux density of $4.73 \mathrm{Jy} \mathrm{K}^{-1}$ from Young et al. (2011). While the post-starbursts have high CO luminosities for their SFRs, the observed offset does not persist for the denser $\mathrm{HCN}$-traced gas; their $L^{\prime}(\mathrm{HCN})$ upper limits are consistent with their low SFRs. The $L^{\prime}(\mathrm{HCN}) / L^{\prime}(\mathrm{CO})$ ratios for post-starbursts are low compared to the star-forming galaxies and most of the CO-detected early types.

Similarly, we compare the $\mathrm{HCO}^{+}$upper limits to the SFRs for the post-starburst targets in Figure 3. The comparison samples of star-forming and starbursting galaxies are from Graciá-Carpio et al. (2008) and the early-type galaxies from Crocker et al. (2012). Again, the upper limits from the poststarburst targets are consistent with their quiescent SFRs, though the limits on $\mathrm{H} 02$ are higher than most of the quiescent comparison sample.

As discussed in Section 2.2, the SFR for H02 is uncertain, with various tracers estimating $\sim 0.09-1 M_{\odot} \mathrm{yr}^{-1}$. In Figures 2 and 3, we see that even at an SFR of $1 M_{\odot} \mathrm{yr}^{-1}, \mathrm{H} 02$ still has a high $L^{\prime}(\mathrm{CO})$ for its SFR, and yet a $L^{\prime}(\mathrm{HCN})$ and $L^{\prime}\left(\mathrm{HCO}^{+}\right)$ consistent with the relation followed by the comparison galaxies.

In French et al. (2015), we proposed several scenarios for explaining the discrepancy between the high CO luminosity and the low SFR aside from a low CO-traced star formation efficiency. Many are now disfavored given the consistency between the HCN luminosity and SFR. The observed discrepancy is not likely driven by unaccounted for dust 


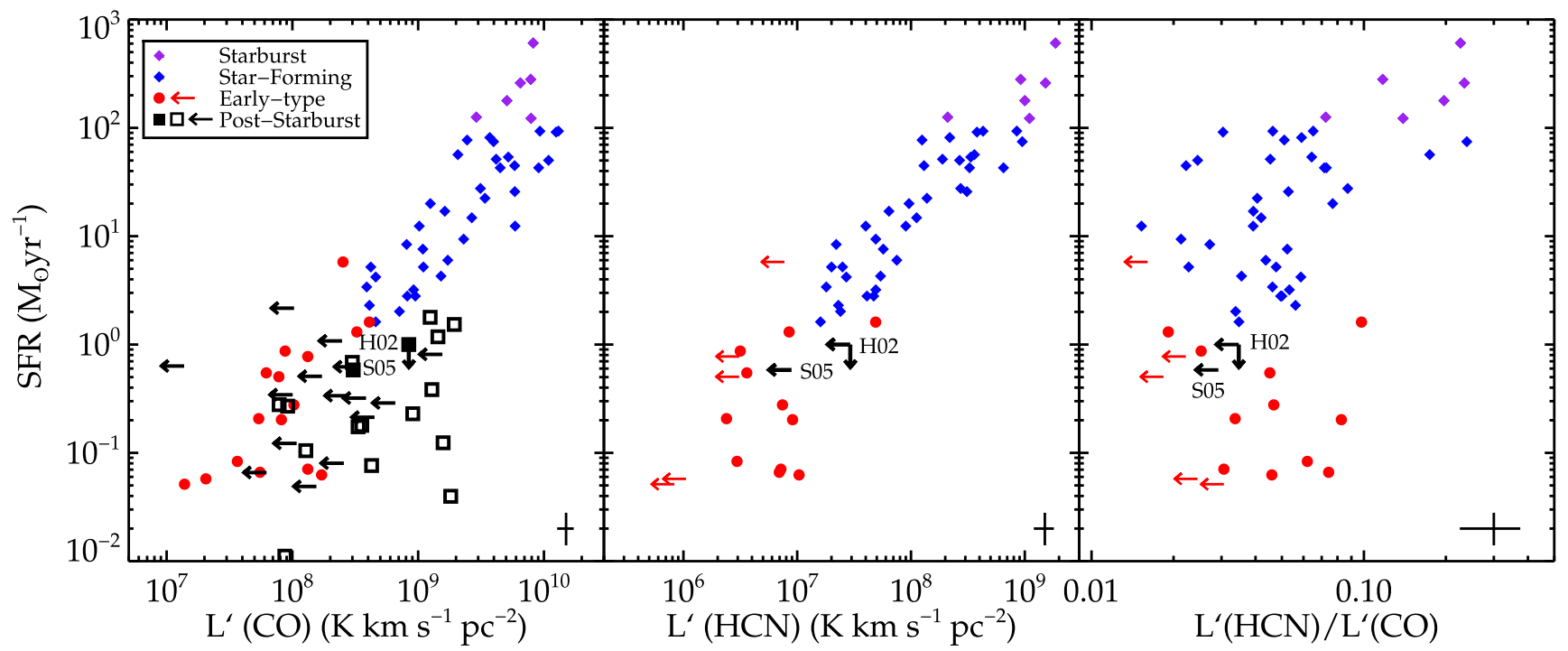

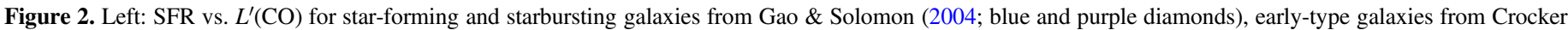

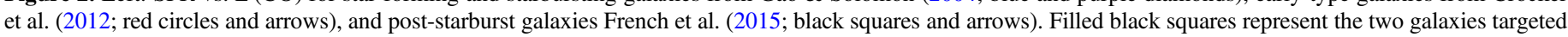

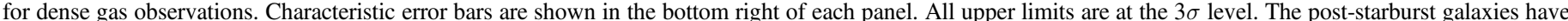

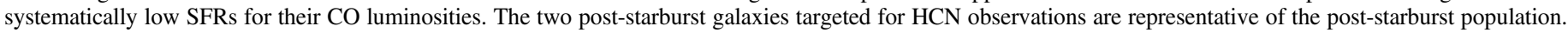

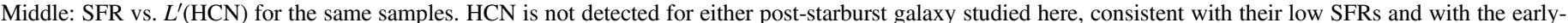

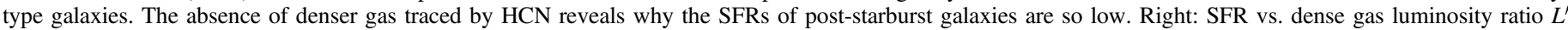

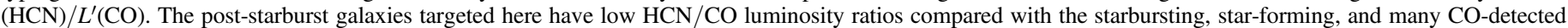

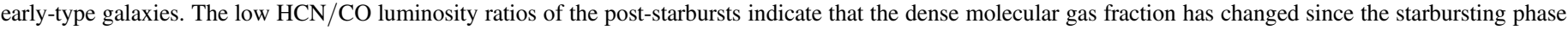
and is different than those in normal star-forming galaxies.

extinction or significant low-mass star formation (a very bottom-heavy IMF) that is hidden from our SFR tracers. It is also improbable that significant star formation is missed in the aperture correction of the SFRs from the $3^{\prime \prime}(2.7-3.2 \mathrm{kpc})$ SDSS fibers (see the discussion in Section 2.2). The range of SFRs inferred from various tracers implies a large range of dust extinction values, and, even so, the $\mathrm{CO}$ luminosities are systematically higher than expected from the low SFRs. Indeed, Smercina et al. (2018) find the SFRs are still much lower than expected given the high $\mathrm{CO}$ luminosities when extinction-insensitive IR-based SFR tracers are used.

The two post-starburst galaxies targeted here have $\mathrm{HCN} / \mathrm{CO}$ luminosity ratios that are low compared to star-forming galaxies and many early types. A similar effect is seen in the $\mathrm{HCO}^{+} / \mathrm{CO}$ luminosity ratios. Here, we compare the poststarburst galaxies to several different galaxy types: early-type (Crocker et al. 2012), star-forming, and starbursting (Gao \& Solomon 2004). We perform a Monte-Carlo analysis, drawing pairs of galaxies at random from these comparison samples, to test how unusual it is to find two galaxies with these $\mathrm{HCN} / \mathrm{CO}$ luminosity ratios. Drawing from the combined sample of earlytype, star-forming, and starbursting galaxies, the probability of finding two galaxies with $\mathrm{HCN} / \mathrm{CO}$ as low as the poststarbursts is $5 \%$. Thus, the post-starbursts have relatively low $\mathrm{HCN} / \mathrm{CO}$ luminosity ratios, and it is unlikely that our observed upper limits are due to random selection. We also compare to the comparison galaxy populations individually, to address several specific questions.

Next we compare to the three galaxy types separately. The starbursting sample represents likely recent progenitors of $\mathrm{H} 02$ and S05. There are zero starbursting (SFR $\geqslant 100 M_{\odot} \mathrm{yr}^{-1}$ ) galaxies with $\mathrm{HCN} / \mathrm{CO}$ ratios as low as the post-starbursts. This large difference indicates that the dense molecular gas fraction in the post-starburst galaxy has changed significantly since the starburst phase. Next, we consider a comparison to
H02 and S05's likely descendants: the early-type sample. Drawing from just the early-type sample, we find that two galaxies have $\mathrm{HCN} / \mathrm{CO}$ luminosity ratios as low as the two post-starbursts $16 \%$ of the time; thus, these observations alone are not sufficient to distinguish the post-starburst sample from the early types. The post-starburst sample may already be consistent with the early-type galaxies, or a separate mechanism could be lowering the dense gas ratio in each (we discuss this further in Section 4.4).

So far, we have connected the lack of dense gas to why poststarburst galaxies are not forming stars. Here we explore why the $\mathrm{HCN} / \mathrm{CO}$ luminosity ratio is low, so we compare to normal star-forming galaxies (SFR $<100 M_{\odot} \mathrm{yr}^{-1}$ ). While the poststarburst galaxies have $\mathrm{CO}$ luminosities similar to this comparison sample, the post-starburst SFRs are lower. Comparing the $\mathrm{HCN} / \mathrm{CO}$ luminosity ratios, the post-starburst galaxies have a lower dense gas fraction. Drawing from the normal star-forming sample, we find two galaxies with HCN/ $\mathrm{CO}$ as low as the post-starbursts only $2 \%$ of the time in our Monte-Carlo analysis. This significant difference in dense molecular gas fractions implies the state of the molecular gas reservoir is substantially different than expected in the course of normal star formation. We discuss several physical mechanisms that could alter the molecular gas state, lowering the dense gas fraction, in Section 4.4.

In summary, post-starburst galaxies have CO luminosities that are systematically high given their SFRs, when compared to the SFR $-L^{\prime}(\mathrm{CO})$ relation that is followed by star-forming and early-type galaxies. However, the low $\mathrm{HCN}$ and $\mathrm{HCO}^{+}$ luminosities implied by our ALMA nondetections are consistent with the low SFRs in the two post-starbursts targeted here. These low dense gas luminosities and SFRs are typical of early-type galaxies, the likely end points of post-starburst evolution. The HCN/CO luminosity ratios are low compared to starbursting, star-forming, and many CO-detected early-type 


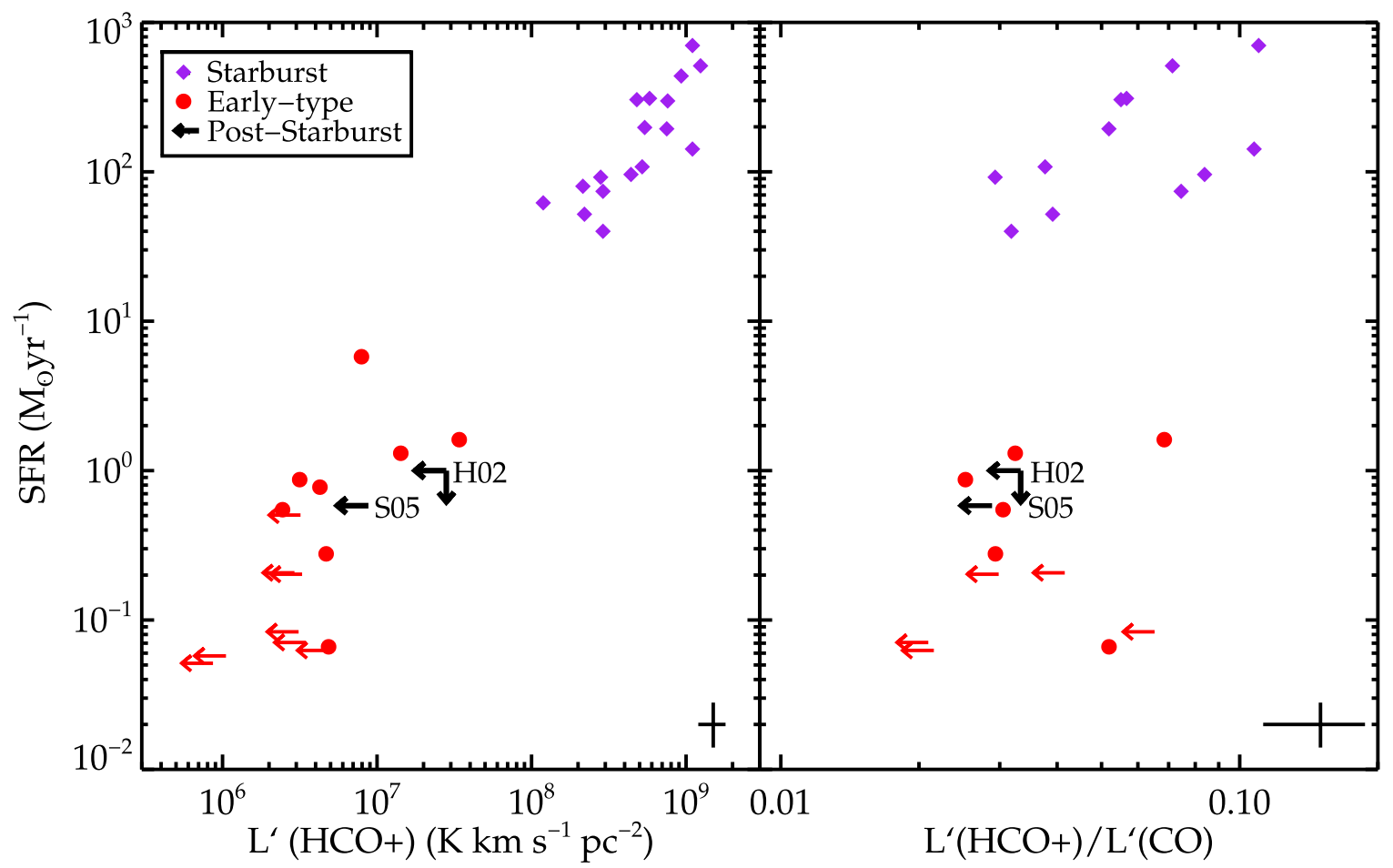

Figure 3. Left: SFR vs. $\mathrm{L}^{\prime}\left(\mathrm{HCO}^{+}\right)$for starbursting galaxies from Graciá-Carpio et al. (2008; purple diamonds), early-type galaxies from Crocker et al. (2012; red circles and arrows), and post-starburst galaxies. All upper limits are at the $3 \sigma$ level. $\mathrm{HCO}^{+}$is not detected for either CO-traced gas-rich post-starburst studied here, which is consistent with expectations from their low SFRs. Right: SFR vs. dense gas luminosity ratio $L^{\prime}\left(\mathrm{HCO}^{+}\right) / L^{\prime}(\mathrm{CO})$. As with the HCN/CO luminosity ratio in Figure 2, the post-starburst galaxies have low $\mathrm{HCO}^{+} / \mathrm{CO}$ luminosity ratios compared with starbursting and many CO-detected early-type galaxies.

galaxies, implying a low fraction of dense molecular gas mass to total molecular gas mass.

\section{Discussion}

\subsection{Interpretation of the $L^{\prime}(H C N)-S F R$ Relation}

Despite the tight linear relation between $\mathrm{HCN}$ luminosity and SFR, there are still several uncertainties in the physical interpretation of this relation. Some have interpreted the fact that the $L^{\prime}(\mathrm{HCN})-\mathrm{SFR}$ relation is more linear and has less scatter than the $L^{\prime}(\mathrm{CO})-\mathrm{SFR}$ relation as evidence of a threshold density for star formation (Wu et al. 2005; Heiderman et al. 2010; Lada et al. 2012). However, the difference between these two relations can also be explained by whether the median gas density is above or below the critical density of CO (1-0) or HCN (1-0) (Krumholz \& Thompson 2007), and the subsequent relation between the gas density and line luminosity given the emission from subthermal gas (Narayanan et al. 2008). Furthermore, Stephens et al. (2016) find that the kiloparsec-scale observations of integrated galaxy properties cannot be explained by a simple summation of clumps and suggest that the low scatter in the $L^{\prime}(\mathrm{HCN})-\mathrm{SFR}$ relation is due to a universal dense gas star formation efficiency, universal stellar IMF, and universal core/clump mass functions, with the kiloparsec scale being sufficient to sample the full mass functions as well as various evolutionary states. Thus, we do not necessarily expect the $\mathrm{HCN}$-traced gas to trace gas where collapse to stars is inevitable, and such a threshold is likely much higher than $10^{4} \mathrm{~cm}^{-3}$ (Krumholz \& Tan 2007). Nevertheless, despite potential nuances in the interpretation of the linear SFR-HCN relations, the $\mathrm{HCN} / \mathrm{CO}$ ratio serves as a proxy for the dense gas fraction.

\subsection{Dense Gas in Other Post-starburst-like Galaxies}

There are two other post-starburst-like galaxies with dense gas observations in the literature. We do not include them in our analysis because they are not selected by our post-starburst selection criteria, but we discuss them here. The first is NGC 5195 (M51b). While the nucleus of this galaxy shows a poststarburst signature (spectrum from Heckman et al. 1980), the integrated spectrum (Kennicutt 1992) does not have the significant Balmer absorption required to be selected into our sample. This galaxy was observed in $\mathrm{CO}(1-0)$ and $\mathrm{HCN}(1-0)$ by Kohno et al. (2002) with the Nobeyama $45 \mathrm{~m}$. Subsequent observations by Matsushita et al. (2010) did not detect HCN. Observations of NGC 5195 are complicated by the nearby spiral arm of M51 in this interacting system. Resolved measurements by Kohno et al. (2002) using the Nobeyama Millimeter Array are brighter than the $15^{\prime \prime}$ beam unresolved observations. Alatalo et al. (2016a) reobserved this galaxy using the Combined Array for Research in Millimeter Astronomy and found a CO (1-0) line flux in between the two Kohno et al. (2002) measurements. NGC 5195 has both HCN and CO luminosities consistent with its SFR from Lanz et al. (2013), unlike our targets. Alatalo et al. (2016a) conclude that this galaxy has a star formation efficiency consistent with normal early-type galaxies.

Another galaxy with some post-starburst characteristics and dense gas measurements is NGC 1266 (Alatalo et al. 2014, 2015). HCN (1-0) and CO (1-0) measurements are part of the Atlas-3D survey of early-type galaxies (Crocker et al. 2012). While this galaxy has nebular emission lines that would exclude it from our post-starburst sample, it is possible that this "shocked" post-starburst galaxy is a precursor to our sample (Alatalo et al. 2016b). NGC 1266 has the highest $\mathrm{HCN} / \mathrm{CO}$ 
line luminosity ratio of any of the galaxies in the Crocker et al. (2012) sample, in contrast to the low dense gas ratios seen here. Alatalo et al. (2015) claim that this difference may be caused by the molecular outflow in the nucleus of this galaxy enhancing the dense gas fraction in this region. NGC 1266 also has a unusually excited high- $J$ CO emission, possibly from shocks (Pellegrini et al. 2013). In these integrated measurements, NGC 1266 appears to have a low SFR $/ L^{\prime}(\mathrm{HCN})$ and a normal $\mathrm{SFR} / L^{\prime}(\mathrm{CO})$. However, spatially resolved measurements show the galaxy to have a low SFR $/ L^{\prime}(\mathrm{CO})$ surface density ratio, perhaps driven by the galaxy's outskirts, with a normal SFR $/ L^{\prime}(\mathrm{CO})$ in its nucleus (Alatalo et al. 2015).

\subsection{Variations in Luminosity to Mass Conversion}

In order to interpret the $\mathrm{HCN}$ and $\mathrm{CO}$ luminosities as tracers of molecular gas mass, we must use conversion factors to convert $L^{\prime}(\mathrm{HCN})$ to a dense $\left(n \gtrsim 8 \times 10^{3} \mathrm{~cm}^{-3}\right)$ molecular gas mass upper limit and $L^{\prime}(\mathrm{HCN}) / L^{\prime}(\mathrm{CO})$ into the dense molecular gas mass to total molecular gas mass ratio (i.e., the "dense gas mass fraction"). We assume that the conversion factor from $L^{\prime}(\mathrm{HCN})$ to dense molecular gas mass is the same for post-starbursts and other galaxies. Therefore, our nondetections of $L^{\prime}(\mathrm{HCN})$ imply low dense gas masses, relative to the dense gas mass-SFR relation traced by star-forming and earlytype galaxies, explaining their quiescence.

We test whether the dense gas mass ratio could be normal, despite the observed dense gas luminosity ratio, due to uncertainties in the conversion factors. What if $L^{\prime}(\mathrm{HCN}) / L^{\prime}$ (CO) does not correlate with the dense gas mass fraction as it does for other galaxies? This scenario could occur if the $\mathrm{CO}$ luminosity to total molecular gas mass conversion factor $\alpha_{\mathrm{CO}}$ and HCN luminosity to dense molecular gas mass conversion factor $\alpha_{\mathrm{HCN}}$ vary differently with the state of the gas.

A lower value of $\alpha_{\mathrm{CO}}$ is usually invoked in ultra luminous infrared galaxies (ULIRGs) and justified based on differences in the distribution or state of the gas, widening the linewidth (e.g., Downes \& Solomon 1998; Narayanan et al. 2012). In French et al. (2015), we consider whether a ULIRG-like $\alpha_{\mathrm{CO}}$ may be appropriate for post-starburst galaxies. While poststarbursts may be descendants of ULIRGs, we are observing them many dynamical times $\left(\sim 10^{6}-10^{7.5} \mathrm{yr}\right.$, Genzel et al. 2010) after the starburst phase has ended ( 0.3-1 Gyr ago). We estimate the influence of the stellar potential on increasing the linewidth and lowering $\alpha_{\mathrm{CO}}$, and find that it is not sufficient to resolve the observed offset between $L^{\prime}(\mathrm{CO})$ and the low SFRs. Additionally, Smercina et al. (2018) find gas to dust ratios in post-starburst galaxies consistent with nearby galaxies using a Milky Way-like value of $\alpha_{\mathrm{CO}}$, indicating that significantly lower conversion factors are unlikely. The dense gas conversion factor is similarly uncertain, but to resolve the discrepancies for the post-starburst galaxies in both SFR- $L^{\prime}$ (CO) and SFR- $L^{\prime}(\mathrm{HCN})$, some other effect would have to lower $\alpha_{\mathrm{CO}}$ without a decrease in $\alpha_{\mathrm{HCN}}$.

We test this possibility using DESPOTIC (Krumholz 2013) to model the change in $\alpha_{\mathrm{CO}}$ and $\alpha_{\mathrm{HCN}}$ for the typical Milky Way GMC and ULIRG conditions described by Krumholz (2013). ULIRG conditions result in $\alpha_{\text {CO }}$ values $\sim 5 \times$ lower than those in Milky Way GMC conditions, as expected. However, $\alpha_{\mathrm{HCN}}$ is lowered by the same factor. Thus, even ULIRG-like conditions could not generate the low observed $\mathrm{HCN} / \mathrm{CO}$ luminosity ratios of post-starburst galaxies. The low
$\mathrm{HCN} / \mathrm{CO}$ luminosity ratios in the post-starburst targets are thus likely due to low dense gas mass ratios.

\subsection{What Prevents the CO-traced Gas from Further Collapse?}

The low observed upper limits on the HCN-traced gas present a puzzle when coupled with the observations of significant CO-traced gas: what prevents the CO-traced gas from further collapse to denser gas? In order to explain the low dense gas mass ratios, some mechanism must prevent collapse of the CO-traced gas, affecting the ability of dense gas to form. Here, we explore possible mechanisms to prevent this collapse.

One possibility for inhibiting collapse is if kinetic energy is injected into the gas, rendering it stable from gravitational collapse. An example of this process is "morphological quenching" (Martig et al. 2009, 2013), where the increased shear in early-type galaxies stabilizes the gas from gravitational collapse, decreasing the dense gas fraction and inhibiting star formation. We note that, while the post-starbursts have a low dense gas fraction compared to the full set of comparison galaxies we consider, they may not have an especially low dense gas fraction compared to early-type galaxies. If morphological quenching is already lowering the dense gas fraction in early-type galaxies compared to star-forming and starbursting galaxies, this would explain why we do not see a significant difference in the dense gas fractions of the poststarburst and early-type galaxies.

Another possible energy source is the dissipation of turbulence from AGN jets or shocks (Nesvadba et al. 2010). However, the role of turbulent energy is complex, even in "normal" star-forming galaxies, simultaneously increasing the dense gas fraction by driving collapse at small scales, and suppressing star formation by preventing the collapse of GMCs on larger scales (e.g., Federrath \& Klessen 2013). Guillard et al. (2015) suggest that heating from the dissipation of AGNinjected turbulence inhibits gravitational collapse on all scales as it cascades.

Conversely, turbulence from stellar feedback is invoked to explain the high gas densities and high dense gas fractions in ULIRGs (e.g., Papadopoulos et al. 2012; Hopkins et al. 2013). More detailed modeling of the gas state in this unusual suppressed state is needed to understand how various sources of kinetic energy can act to lower the dense gas fraction in these galaxies.

What then is the physical source of the injected energy? Many dynamical times have passed since the starburst ended, so energy or turbulence from stellar feedback is unlikely. Secular sources of turbulence like morphological quenching act over $>1 \mathrm{Gyr}$, and thus are unlikely to play a role during this short transitional phase.

We have selected the post-starburst galaxies on a lack of emission lines, which selects against strong AGNs. Nevertheless, LINER-like activity is currently seen in many poststarburst galaxies (Yan et al. 2006; Yang et al. 2006; French et al. 2015). Obscured AGN activity in a small number of sources, including $\mathrm{H} 02$, is suggested by the dust spectral fitting done by Smercina et al. (2018). Smercina et al. also observe $\mathrm{H}_{2}$ rotational lines in S05 in the mid-IR, satisfying the turbulent heating threshold of $\mathrm{H}_{2} / 7.7 \mu \mathrm{m}>0.04$ seen in molecular hydrogen emission-line galaxies (Ogle et al. 2010). Further evidence of AGN activity affecting the molecular gas reservoirs is seen in the rapid decline of the CO-traced gas during the 
post-starburst phase, on a timescale too rapid to be explained by star formation (French et al. 2018), with timescales and inferred outflow rates similar to those seen in LINERs (Cicone et al. 2014).

We also may be observing the post-starburst galaxies in the nonactive phase of a trend of AGN variability. AGN activity is possible during the post-starburst phase (Davies et al. 2007; Schawinski et al. 2009; Wild et al. 2010; Cales \& Brotherton 2015; Baron et al. 2017), and varies with timescales short enough to observe turbulent gas without an active AGN. AGNs are seen to turn on and off with timescales of $\sim 10^{4}-10^{5}$ years (Lintott et al. 2009; Keel et al. 2017), and the timescale for turbulent energy from an AGN to deplete is $\sim 10^{7}-10^{8}$ years (Guillard et al. 2015). One possible example of this is seen by Prieto et al. (2016), who observe light echoes from past AGN activity in a post-starburst galaxy with a LINER-like center. AGN activity is also observed to suppress star formation in CO-traced gas (Ho 2005; Nesvadba et al. 2011; Guillard et al. 2015; Lanz et al. 2016).

Thus, while the details of how kinetic energy injected into the gas might lower the low dense gas fraction are still not understood, these post-starburst galaxies may have experienced recent AGN activity strong enough to disrupt the molecular gas and suppress star formation.

\subsection{Evolution to Early-type Galaxies}

The presence of large CO-traced molecular gas reservoirs in half of the post-starbursts studied also presents a puzzle in understanding how these post-starburst galaxies can evolve to normal early-type galaxies. Post-starburst galaxies have stellar populations, color gradients, morphologies, and kinematics consistent with reaching the red sequence of early-type galaxies (Norton et al. 2001; Yang et al. 2004, 2008; Pracy et al. 2013; Pawlik et al. 2015) in a few gigayears. However, early-type galaxies are typically gas poor, with molecular gas fractions of $\lesssim 10^{-3}$ (Young et al. 2011). What is the fate of the CO-traced gas reservoirs in these post-starburst galaxies? We find in French et al. (2018) that the CO-traced molecular gas to stellar mass fraction declines with the time elapsed since the starburst ended, implying that post-starburst galaxies should reach earlytype levels of molecular gas in 700-1500 Myr. Thus, poststarbursts become gas poor as their stellar populations, color gradients, morphologies, and kinematics start to resemble early types.

The CO-traced gas undergoes a dramatic transition as the galaxy evolves from the starbursting to the post-starburst phases over $\lesssim 1$ Gyr. Starburst galaxies have enhanced COtraced SFEs compared to normal star-forming galaxies. After the starburst ends, we observe post-starburst galaxies to have suppressed CO-traced SFEs relative to normal star-forming galaxies. Over the $\sim$ gigayear of evolution between these two phases, the dense gas mass ratio also evolves from high to low, but the HCN luminosity tracks with the SFR throughout this process. Thus, despite the fact that collapse to star formation is not guaranteed at the $n \gtrsim 8 \times 10^{3} \mathrm{~cm}^{-3}$ densities traced by $\mathrm{HCN}$, the processes that drive the starburst, the end of the starburst, and the dramatic change in CO-traced SFEs do not affect the $L^{\prime}(\mathrm{HCN})-\mathrm{SFR}$ relation. This result is consistent with the idea proposed by Krumholz \& Thompson (2007) and Stephens et al. (2016) that the dense gas SFE is universal on kiloparsec scales. While these studies were based on star-forming and starbursting galaxies, our result suggests that this universality may extend to quiescent galaxies with low SFRs.

\section{Conclusions}

We survey the dense molecular gas content of two poststarburst galaxies possessing large reservoirs of CO-traced molecular gas, despite their lack of significant current star formation. ALMA does not detect either $\mathrm{HCN}(1-0)$ or $\mathrm{HCO}^{+}$ $(1-0)$ in these galaxies. This absence of denser gas is consistent with their low SFRs. For the first time, we have direct evidence as to why post-starburst galaxies are now quiescent: the denser gas required for star formation is absent. The $\mathrm{HCN} / \mathrm{CO}$ luminosity ratio upper limits are low compared to star-forming and many CO-detected early-type galaxies, implying a low fraction of dense molecular gas mass to total molecular gas mass.

The low HCN luminosities of the post-starburst galaxies are already consistent with the early-type galaxies into which they are expected to evolve. However, the significant $\mathrm{CO}$-traced gas and thus the low dense gas fraction necessitates a more detailed view of how these galaxies could evolve into gas poor early types, and what prevents the CO-traced gas from collapsing further. The ( $200 \mathrm{Myr})$ decline in the CO-traced molecular gas during the post-starburst phase (French et al. 2018) is too rapid to be explained by star formation alone. Thus, any successful feedback model must predict that both the $\mathrm{CO}$ traced gas declines over this rapid timescale and that the $\mathrm{CO}$ traced gas is stable against collapse to denser gas, possibly via the same mechanism.

This picture of how star formation ends and the molecular gas reservoirs are depleted in galaxies undergoing rapid transitions may be largely representative, as $\sim 40 \%-100 \%$ of galaxies are expected to evolve through this phase (Zabludoff et al. 1996; Snyder et al. 2011; Wild et al. 2016), and higher redshift post-starbursts are also observed to have large COtraced molecular gas reservoirs (Suess et al. 2017).

K.D.F. is supported by Hubble Fellowship Grant HST-HF251391.001-A, provided by NASA through a grant from the Space Telescope Science Institute, which is operated by the Association of Universities for Research in Astronomy, Incorporated, under NASA contract NAS5-26555. A.I.Z. acknowledges funding from NASA grant ADP-NNX10AE88G. D.N. acknowledges support from grants NSF AST-1724864, AST-1715206, and HST AR-13906,15043. A.S. acknowledges support for this work by the National Science Foundation Graduate Research Fellowship Program under grant No. DGE 1256260. Any opinions, findings, and conclusions or recommendations expressed in this material are those of the author(s) and do not necessarily reflect the views of the National Science Foundation.

This paper makes use of the following ALMA data: ADS/ JAO.ALMA\#2016.1.00881.S. ALMA is a partnership of ESO (representing its member states), NSF (USA), and NINS (Japan), together with NRC (Canada), NSC and ASIAA (Taiwan), and KASI (Republic of Korea), in cooperation with the Republic of Chile. The Joint ALMA Observatory is operated by ESO, AUI/NRAO, and NAOJ. Funding for SDSS-III has been provided by the Alfred P. Sloan Foundation, the Participating Institutions, the National Science Foundation, and the U.S. Department of Energy Office of Science. The SDSS-III website is http://www.sdss3.org/. 


\section{ORCID iDs}

K. Decker French (1) https://orcid.org/0000-0002-4235-7337 Ann I. Zabludoff (1) https://orcid.org/0000-0001-6047-8469 Ilsang Yoon (ib https://orcid.org/0000-0001-9163-0064 Yujin Yang (i) https://orcid.org/0000-0003-3078-2763 Adam Smercina (1) https://orcid.org/0000-0003-2599-7524 J. D. Smith (1) https://orcid.org/0000-0003-1545-5078 Desika Narayanan (1) https://orcid.org/0000-0002-7064-4309

\section{References}

Aalto, S., Garcia-Burillo, S., Muller, S., et al. 2012, A\&A, 537, 44 Alatalo, K., Aladro, R., Nyland, K., et al. 2016a, ApJ, 830, 137 Alatalo, K., Cales, S. L., Rich, J. A., et al. 2016b, ApJS, 224, 38 Alatalo, K., Lacy, M., Lanz, L., et al. 2015, ApJ, 798, 31 Alatalo, K., Lisenfeld, U., Lanz, L., et al. 2016c, ApJ, 827, 106 Alatalo, K., Nyland, K., Graves, G., et al. 2014, ApJ, 780, 11 Baron, D., Netzer, H., Poznanski, D., et al. 2017, MNRAS, 470, 1687 Becker, R. H., White, R. L., \& Helfand, D. J. 1995, ApJ, 450, 559 Cales, S. L., \& Brotherton, M. S. 2015, MNRAS, 449, 2374 Chen, H., Gao, Y., Braine, J., \& Gu, Q. 2015, ApJ, 810, 140 Cicone, C., Maiolino, R., Sturm, E., et al. 2014, A\&A, 562, 21 Condon, J. J. 1992, ARA\&A, 30, 575

Couch, W. J., \& Sharples, R. M. 1987, MNRAS, 229, 423

Crocker, A., Krips, M., Bureau, M., et al. 2012, MNRAS, 421, 1298

Davies, R., Sanchez, F. M., Genzel, R., et al. 2007, ApJ, 671, 1388

Davis, T. A., Young, L. M., Crocker, A. F., et al. 2014, MNRAS, 444, 3427 de Vries, W. H., Hodge, J. A., Becker, R. H., White, R. L., \& Helfand, D. J. 2007, AJ, 134, 457

Downes, D., \& Solomon, P. M. 1998, ApJ, 507, 615

Dressler, A., \& Gunn, J. E. 1983, ApJ, 270, 7

Federrath, C., \& Klessen, R. S. 2013, ApJ, 763, 51

French, K. D., Yang, Y., Zabludoff, A., et al. 2015, ApJ, 801, 1

French, K. D., Yang, Y., Zabludoff, A., \& Tremonti, C. 2018, ApJ, in press (arXiv:1806.03301)

Gao, Y., \& Solomon, P. M. 2004, ApJ, 606, 271

Genzel, R., Tacconi, L. J., Gracia-Carpio, J., et al. 2010, MNRAS, 407, 2091 Graciá-Carpio, J., García-Burillo, S., Planesas, P., Fuente, A., \& Usero, A. 2008, A\&A, 479, 703

Guillard, P., Boulanger, F., Lehnert, M. D., et al. 2015, A\&A, 574, A32

Hayward, C. C., Lanz, L., Ashby, M. L. N., et al. 2014, MNRAS, 445, 1598 Heckman, T. M., Crane, P. C., \& Balick, B. 1980, A\&AS, 40, 295

Heiderman, A., Evans, N. J., Allen, L. E., Huard, T., \& Heyer, M. 2010, ApJ, 723,1019

Ho, L. C. 2005, ApJ, 629, 680

Hopkins, P. F., Narayanan, D., Murray, N., \& Quataert, E. 2013, MNRAS, 433, 69

Juneau, S., Narayanan, D. T., Moustakas, J., et al. 2009, ApJ, 707, 1217

Keel, W. C., Lintott, C. J., Maksym, W. P., et al. 2017, ApJ, 835, 256

Kennicutt, J. 1998, ApJ, 498, 541
Kennicutt, R. 1992, ApJS, 79, 255

Kepley, A. A., Leroy, A. K., Frayer, D., et al. 2014, ApJL, 780, L13

Kohno, K., Tosaki, T., Matsushita, S., et al. 2002, PASJ, 54, 541

Krumholz, M. R. 2013, MNRAS, 437, 1662

Krumholz, M. R., \& Tan, J. C. 2007, ApJ, 654, 304

Krumholz, M. R., \& Thompson, T. A. 2007, ApJ, 669, 289

Lada, C. J., Forbrich, J., Lombardi, M., \& Alves, J. F. 2012, ApJ, 745, 190

Lanz, L., Ogle, P. M., Alatalo, K., et al. 2016, ApJ, 826, 29

Lanz, L., Zezas, A., Brassington, N., et al. 2013, ApJ, 768, 90

Lintott, C. J., Schawinski, K., Keel, W., et al. 2009, MNRAS, 399, 129

Loomis, R. A., Öberg, K. I., Andrews, S. M., et al. 2018, AJ, 155, 182

Martig, M., Bournaud, F., Teyssier, R., \& Dekel, A. 2009, ApJ, 707, 250

Martig, M., Crocker, A. F., Bournaud, F., et al. 2013, MNRAS, 432, 1914

Matsushita, S., Kawabe, R., Kohno, K., Tosaki, T., \& Vila-Vilaro, B. 2010, PASJ, 62, 409

Morić, I., Smolčić, V., Kimball, A., et al. 2010, ApJ, 724, 779

Narayanan, D., Cox, T. J., Kelly, B., et al. 2008, ApJS, 176, 331

Narayanan, D., Krumholz, M. R., Ostriker, E. C., \& Hernquist, L. 2012, MNRAS, 421, 3127

Nesvadba, N. P. H., Boulanger, F., Lehnert, M. D., Guillard, P., \& Salome, P. 2011, A\&A, 536, L5

Nesvadba, N. P. H., Boulanger, F., Salomé, P., et al. 2010, A\&A, 521, A65

Norton, S. A., Gebhardt, K., Zabludoff, A. I., \& Zaritsky, D. 2001, ApJ, 557,150

Ogle, P., Boulanger, F., Guillard, P., et al. 2010, ApJ, 724, 1193

Papadopoulos, P. P., van der Werf, P. P., Xilouris, E. M., et al. 2012, MNRAS, 426, 2601

Pawlik, M. M., Wild, V., Walcher, C. J., et al. 2015, MNRAS, 456, 3032

Pellegrini, E. W., Smith (PI), J. D., Wolfire, M. G., et al. 2013, ApJL, 779, L19

Pracy, M. B., Croom, S., Sadler, E., et al. 2013, MNRAS, 432, 3131

Prieto, J. L., Krühler, T., Anderson, J. P., et al. 2016, ApJL, 830, L32

Rowlands, K., Wild, V., Nesvadba, N., et al. 2015, MNRAS, 448, 258

Salas, P., Galaz, G., Salter, D., et al. 2014, ApJ, 797, 134

Schawinski, K., Lintott, C. J., Thomas, D., et al. 2009, ApJ, 690, 1672

Scoville, N., Sheth, K., Walter, F., et al. 2015, ApJ, 800, 70

Shirley, Y. L. 2015, PASP, 127, 299

Smercina, A., Smith, J. D. T., Dale, D. A., et al. 2018, ApJ, 855, 51

Snyder, G. F., Cox, T. J., Hayward, C. C., Hernquist, L., \& Jonsson, P. 2011, ApJ, 741, 77

Stephens, I. W., Jackson, J. M., Whitaker, J. S., et al. 2016, ApJ, 824, 29

Suess, K. A., Bezanson, R., Spilker, J. S., et al. 2017, ApJL, 846, L14

Wild, V., Almaini, O., Dunlop, J., et al. 2016, MNRAS, 463, 832

Wild, V., Heckman, T., \& Charlot, S. 2010, MNRAS, 405, 933

Wu, J., Evans, N. J., Shirley, Y. L., \& Knez, C. 2010, ApJS, 188, 313

Wu, J., Evans, N. J., II, Gao, Y., et al. 2005, ApJL, 635, L173

Yan, R., Newman, J. A., Faber, S. M., et al. 2006, ApJ, 648, 281

Yang, Y., Tremonti, C. A., Zabludoff, A. I., \& Zaritsky, D. 2006, ApJL, 646, L33

Yang, Y., Zabludoff, A. I., Zaritsky, D., Lauer, T. R., \& Mihos, J. C. 2004, ApJ, 607, 258

Yang, Y., Zabludoff, A. I., Zaritsky, D., \& Mihos, J. C. 2008, ApJ, 688, 945

Young, L. M., Bureau, M., Davis, T. A., et al. 2011, MNRAS, 414, 940

Zabludoff, A. I., Zaritsky, D., Lin, H., et al. 1996, ApJ, 466, 104 